\title{
Alzheimer's - Looking beyond plaques
}

\author{
W. Sue T. Griffin
}

Address: Geriatric Research, Education and Clinical Center, Neurobiology, Physiology, and Psychiatry, University of Arkansas for Medical Sciences, and the Geriatrics, Education Clinical Center, Central Arkansas Veterans Healthcare System, Little Rock, Arkansas 72205, USA

Email: griffinsuet@uams.edu

FI000 Medicine Reports 20II, 3:24 (doi:10.3410/M3-24)

This is an open-access article distributed under the terms of the Creative Commons Attribution-Non Commercial License (http://creativecommons.org/licenses/by-nc/3.0/legalcode), which permits unrestricted use, distribution, and reproduction in any medium, provided the original work is properly cited. You may not use this work for commercial purposes.

The electronic version of this article is the complete one and can be found at: http://f $1000 . c o m / r e p o r t s / m / 3 / 24$

\begin{abstract}
Mounting evidence shows that inflammation plays a critical role in causing Alzheimer's disease. Over the last few decades we have gone from a situation where inflammation was generally believed to have no role in the disease to the current picture where chronic activation of IL-I inflammation has been shown to account for many of the hallmarks of the disease. This review is a personal account of the quest to prove that inflammation plays a critical role in causing Alzheimer's disease.
\end{abstract}

Even today, Alzheimer's is still a disease that is definitively diagnosed only after death and autopsy, when it is easy to recognize the disease's cardinal features: a shrunken brain with amyloid plaques dotted among neurons laden with neurofibrillary tangles, and often with inclusions similar to those found in the brains of patients who have died of Parkinson's. These irrefutable histological markers of Alzheimer's led to the logical conclusion by most researchers that plaques are the cause of the problem. Many pharmaceutical companies have taken vigorous aim at amyloid with no clear evidence so far that ridding the brain of plaques in Alzheimer's disease results in cognitive improvement [1]. Lacking a smoking gun that definitively singles out the plaques as the causative agent, amyloid is the scientific equivalent of a culprit assumed guilty until proven innocent.

A secondary role for plaques in pathogenesis is not a new idea. The first such plaques were observed by Paul Blocq and Georges Marinesco in 1892 in the brain of a patient with epilepsy. They suggested that the source of the plaques was the surrounding small cells (reviewed in [2]) implying that amyloid plaques are markers left after the occurrence of a series of events such as neuronal damage engendered by neuronal hyperexcitability in epilepsy, genetic variations, head trauma, and aging. In 1907, Oskar Fischer almost immediately set aside the importance of the small cells or any other entities other than amyloid plaques as central in Alzheimer's pathogenesis, and the amyloid hypothesis was accepted as the cause of the disease [1].

A hundred years later, in the 1980s, the amyloid-plaque hypothesis gained further ground with the sequencing of the amyloid beta peptide in plaques and the mapping of the gene encoding its precursor protein (APP) to chromosome 21. Identification of a small group of Alzheimer's families that carried mutations in their APP gene more or less solidified amyloid as the important facet in Alzheimer's pathogenesis. However, inheritance of such mutations did not explain the vast majority of Alzheimer's cases-the sporadic, non-genetic cases.

My interest and those of today's new crop of researchers centers on the potential of Blocq and Marinesco's small cells comprised of microglia and astrocytes, now known to constitute the brain's innate immune system. I envisioned that sources that act beneficially in limited settings, such as glia, would in chronic situations be harmful. Seeing Alzheimer's pathogenesis with fresh eyes and finding that overexpressed proinflammatory proteins are the likely instigators of neuropathological changes, including both plaque and tangle formation, we proposed that amyloid plaques are more likely to be a response to the disease, rather than its initiator, which is an idea that continues to gain acceptance. 


\section{From Down's syndrome to Alzheimer's}

My involvement with Alzheimer's research began somewhat accidentally in 1983 when I attended a seminar on the disease given by Roger Rosenberg at Southwestern Medical School. At the time, I was studying the differentiation of neurons in the developing cerebellum, using a systemic immune disease-the graft-versus-host response-that could be induced, and a few days later "cured." The immune response would temporarily halt neuronal development, allowing me to manipulate specific developmental events in each of the cerebellar cell types [3-5]. Back then, prominent immunologists believed that the immune system and the CNS were completely independent [6], but my observations of the developing rat brain convinced me that there was a connection.

So when Rosenberg showed silver-stained sections from the brains of Alzheimer's patients - enlarged, activated microglia and astrocytes lying among the neuronsamyloid plaques were clearly evident. I couldn't help wondering if those microglia located in and around trash-like plaques, and among neurons that had to exist in their presence, would respond as their macrophage counterparts do when challenged in the periphery. My question was, would they make and release interleukin-1 (IL-1)? I bet yes.

At the time, researchers knew that microglia could act like macrophages and served as the immune cells of the brain. However, back in 1983, all we knew was that they resembled macrophages, the peripheral immune cells that engulf pathogens and secrete the cytokine IL-1, activating multiple immune functions of T helper cells. By then we knew too that the overexpression of IL- 1 in arthritis led to progressive joint deterioration [7]. I wondered whether microglia in the brain might also overexpress IL-1 in Alzheimer's and lead to neuronal deterioration in the brain.

What if damaged or stressed neurons were activating microglia to release excessive amounts of IL-1, which in turn activated astrocytes (analogous to macrophage IL-1 activation of $\mathrm{T}$ helper cells) and caused them to release S100, a soluble astrocyte cytokine known to promote neuron survival? I tested this idea by measuring tissue levels and cellular expression of IL-1 and S100 in brains of patients who had succumbed to Alzheimer's disease and in the brains of disease-free individuals. Indeed, we could see activated glia as well as measure profuse overexpression of IL-1 and S100. It was the first time that a meaningful immune response was shown in the brain. In addition, for the first time inflammatory cytokines such as IL-1 and S100 were associated with Alzheimer's disease. These results laid the ground work for establishing cytokines as contributors to and promoters of neurodegenerative diseases in analogy to their role as drivers in systemic degenerative diseases.

My hypothesis was that, in the early stages, the disease progressed via self-propagating neuronal injury or stress driven by glial activation and cytokine release. Because we had no way of detecting and analyzing early-stage Alzheimer's disease, I needed a model that would mimic the early-onset form of the disease. For this I turned to Down's syndrome. Down's with its three, rather than the usual two, copies of $\beta A P P$ was shown by Henry Wisniewski, at the New York State Institute for Basic Research in Developmental Disabilities, to lead to the clinical and pathological features of Alzheimer's by early middle age [8]. Not only that, but Rachael Neve at Harvard Medical School and her colleagues saw that rather than the 1.5-fold increase in BAPP expected from the presence of triplicate copies of the gene, brain tissue from Down's fetuses had 8 times that in brain from normal fetuses $[8,9]$.

When we examined sections from Down's syndrome brain, we saw that many microglia and astrocytes were enlarged (activated) and overexpressing IL- 1 and S100 in Down's fetuses and newborns. The appearance of these activated glia that were overexpressing these cytokines was present years before plaques were noted in Down's syndrome. These findings supported the idea that glial activation and cytokine expression are the result of neuronal stress, not the presence of amyloid plaques. The neuronal stress in Down's was assumed created by the excess expression of $\beta A P P$ due to its gene duplication from trisomy 21 . We envisioned that the stressed neurons in both Alzheimer's and Down's released something that alerted glia to their distress and their response was activation and increased expression of cytokines. Several years later Steve Barger showed that a fragment of $\beta A P P$, which is secreted in excess from stressed neurons, activates microglia and induces excess release of IL- 1 . Taken together, our studies provided two firsts: the first evidence from the brain of a productive immune response, that is a response to a neuronal stress which precipitates a glial response that then has a neuronal consequence; and the first evidence of the involvement of the brain's immune response in Alzheimer's. The more outlandish idea that I drew from these two firsts was that neuronal stress, without regard to its origin, would elicit a cascade that involved an acute phase neuronal response that included excess expression of $\beta A P P$, release of sAPP, and glial activation with release of IL-1.

By carefully mapping the density of plaques in slices of diseased brain, we discerned a pattern showing that early 
plaques, those that are dispersed rather than dense, are surrounded by a multitude of microglia and astrocytes expressing IL-1 and S100. On the other hand, the denser plaques that appear later had fewer activated glia, suggesting that glial activation and cytokine expression play an important role early in the disease, thus providing further evidence that cytokines could be, in fact, the driving factor [10-12].

While reports from other labs showed that microglia produce IL-1 for activation of astrocytes $[13,14]$ and that S100 is essential for neuronal development and neuronal repair [15], few journal editors in the mid-1980s shared my view that IL-1 and S100 were drivers of Alzheimer's disease progression. Although our work began in 1984, it wasn't published until 1989, when I met Dmitry Goldgaber, who was working at NIH with Carleton Gajdusek on sequencing and mapping BAPP. I met Dmitry at a meeting where I was reporting our findings about Alzheimer's and Down's syndrome. To my surprise, he came to the meeting to report that IL-1 induces synthesis of $\beta A P P$ in cord blood cells. This was a great stroke of luck for both of us. His results offered molecular evidence of a connection between IL-1 and Alzheimer's pathogenesis, which supported my findings about both Alzheimer's and Down's, and our results gave his work a somewhat deeper meaning: if this cytokine was activating the production of BAPP in cord blood, it could be behaving similarly in the brain. Together our studies added credence to the idea that neuronal stress and excess inflammatory cytokine production was a driving force in neurodegeneration and in the production of amyloid plaques. We published our papers back to back in 1989 [16,17].

\section{Tying the tangles together}

Though I had fully expected to return to my work on neuronal development, our proposition that inflammatory cytokines were involved in - and probably driving neurodegeneration was met with such vigorous criticism that I decided to devote more time to the topic.

We followed up our initial studies by examining all of the Alzheimer's-related events for a connection to IL-1. Toward this, we examined the tau protein, a constituent of the tangles seen in Alzheimer's, and the Parkinson'sassociated $\alpha$-synuclein (responsible for producing tangles called Lewy bodies in that disease), both of which were discovered to be associated with Alzheimer's by Virginia Lee and John Trojanowski from the University of Pennsylvania. Tau is a protein that normally stabilizes microtubules, but when it is hyperphosphorylated at multiple sites, as in Alzheimer's, it creates the tangles of filaments, the neurofibrillary tangles in neurons, which are present in an Alzheimer's brain. The protein $\alpha$-synuclein, which forms Lewy bodies in Parkinson's, is actually a fragment of the APP and, though it is present in many cells in the brain, its normal function is still debated. But like other neuropathological changes associated with overexpression of IL-1 in Alzheimer's, the synthesis of $\alpha$-synuclein is also induced by IL-1.

To study the involvement of the tau protein, we implanted slow-release IL-1-containing pellets in the brains of rats and found a twofold increase in tissue levels of total tau mRNA compared to rats implanted with untreated pellets. But since tau does not form neurofibrillary tangles unless hyperphosphorylated, we measured tissue and cellular levels of the hyperphosphorylated form of tau, which is associated with the tangles, and found it was elevated by three fold in rats with IL-1-containing pellets. When we looked at the activity of MAP kinase p38 (MAPK p38) to see if it might be involved in IL-1 induction of tau hyperphosphorylation, we found that IL-1 induces elevation of the MAPK p38 mRNA. What's more, MAPK p38 activity was necessary for tau phosphorylation in both rats imbalanced with IL-1 pellets and in humans. When we stained brain sections from Alzheimer's patients we also saw abundant MAPK p38 in the same neurons that had high levels of hyperphosphorylated tau protein [18]. Now we could say that IL-1 was driving both the production of tau protein, and its hyperphosphorylation, via IL-1 induction of a specific kinase, MAPK p38. To investigate whether IL-1 might also play a role in Lewy body pathology seen in Alzheimer's, we tested the role of IL-1 in $\alpha$-synuclein fiber production using three systems: tissue culture, IL-1-pellet-implanted rat brains, and brain slices from Alzheimer's patients. All three approaches gave the same results, showing that IL-1 overexpression was associated with increased production of $\alpha$-synuclein [19].

George Siggins and colleagues at the Scripps Research Institute had reported that high levels of IL-1 might reduce learning and neurotransmission, so we examined the possibility that IL-1 might contribute to the decrease in the amount of acetylcholine neurotransmitter that is seen in Alzheimer's patients. Toward this, we used our IL-1 pellet experimental paradigm to show that IL-1 elevated both the levels and the activity of the enzyme acetylcholinesterase, which degrades acetylcholine, potentially explaining how the cytokine might be involved in the Alzheimer's-related reduction of this memory-related neurotransmitter [20].

We also investigated whether IL-1 inflammatory pathways were triggered by neuronal damage initiated by sources such as aging, head trauma, epilepsy, and AIDS. Indeed, aging and each of these other conditions put those affected at increased risk for the development of Alzheimer's and all 
are characterized by glial activation and increased IL-1 production.

As a way to explain the inexorable progress of neurodegenerative changes in conditions associated with precocious development of Alzheimer's, I began to think about neuronal stress-induced IL-1 excess as part of a cytokine feed-back cycle, with the initiating insult coming from a variety of sources: a genetic predisposition (such as the APOE $\varepsilon 4$ allele - a risk factor for Alzheimer's), repeated injury (head trauma or epilepsy) or infection (HIV), and aging. Although the inflammatory mechanisms probably help to clear damaged cells in limited situations, in the long term, if microglia are activated to produce IL-1 in a chronic fashion, they can turn on a cycle that leads to more neuronal damage and death: damaged neurons activate IL1-producing microglia which in turn activate the production of BAPP and release of sAPP. This secreted fragment activates microglia to produce more IL-1, stimulating other neuronal responses including tau hyperphosphorylation, plaque formation, and alpha synuclein production.

In neuroinflammation, especially IL-1, we had a viable suspect—and a potential therapeutic target—which everyone seemed to be ignoring. It was recognized that in systemic conditions, such as swollen joints, both the swelling and the IL-1 levels were amenable to nonsteroidal anti-inflammatory drugs such as aspirin and ibuprofen. A crucial study by John Breitner at Johns Hopkins showed that among genetically predisposed identical twin pairs who did not show identical patterns of Alzheimer's onset [20], use of nonsteroidal antiinflammatory drugs (NSAID) by one of the twins was associated with a several-year delay in the onset of Alzheimer's [11]. This was huge. If Alzheimer's could be delayed for up to five years as Breitner showed, the societal and economic gains would be inestimable. Breitner's was not an isolated study; a year later, a Dutch study comparing a large group of Alzheimer's patients who were taking NSAIDs for other ailments versus those who were not showed that taking NSAIDs reduced risk or delayed onset of Alzheimer's disease similar to Breitner's results. These findings encouraged further epidemiological studies, and in 2008 a report from the Department of Veterans Affairs Database showed that when data from 50,000 Alzheimer's patients were compared to those of 200,000 control patients, taking ibuprofen for as long as five years was associated with an almost 50\% reduction in risk for Alzheimer's (as calculated by the overall risk per year of life) [22]. This was followed by a prospective clinical trial of NSAID drugs, including naproxen and celecoxib. However, the study was stopped after about 24 months because of drug safety concerns. However, observations that continued for two or more years revealed a protective effect against the onset of Alzheimer's disease in cognitively normal participants who took naproxen [23].

Much progress has been made, some by us and a lot by others, since the days when researchers believed that Alzheimer's could be understood and explained without invoking a role for the innate immune players in the central nervous system - the glia and their cytokines. Not only is there much evidence that these entities and neuroinflammation play a critical role in Alzheimer's, neuroinflammation has become a topic in its own right.

\section{Abbreviations}

IL-1, interleukin-1; NSAID, non steroidal anti-inflammatory drug; $\beta A P P, \beta$-amyloid precursor protein; MAPK p38, MAP kinase $\mathrm{p} 38$.

\section{Competing interest}

The author declares that she has no competing interests.

\section{References}

I. Holmes C, Boche D, Wilkinson D, Yadegarfar G, Hopkins V, Bayer A, Jones RW, Bullock R, Love S, Neal JW, Zotova E, Nicoll JA: Longterm effects of Abeta42 immunisation in Alzheimer's disease: follow-up of a randomised, placebo-controlled phase I trial. Lancet 2008, 372:2 I6-23.

FI000 factor 9

Evaluated by W Sue Griffin I8 Nov 20I I, Mark A Smith 0I Sep 2008

2. Eikelenboom P, van Exel E, Hoozemans JJ, Veerhuis R, Rozemuller AJ, van Gool WA: Neuroinflammation - an early event in both the history and pathogenesis of Alzheimer's disease. Neurodegener Dis 2010, 7:38-4I.

3. Griffin WS, Crom EN, Head JR: Manipulation of brain DNA synthesis is achieved by using a systemic immunological disease. Proc Natl Acad Sci U S A 1982, 79:4783-5.

4. Griffin WS, Head JR, Pardue S, Morrison MR: Changes in RNA synthesis and messenger RNA content in the cerebellum of rats with graft versus host disease. I Neurochem 1980, 35:880-8.

5. Griffin WS, Head JR, Woodward DJ, Carrol C: Graft-versus-host disease impairs cerebellar growth. Nature 1978, 275:315-7.

6. Griffin WS, TaL L: GVHD: A model for studying the neural regulation of immune function. Transplant Proc 1987, 19: I I 30.

7. Wood DD, Ihrie EJ, Dinarello CA, Cohen PL: Isolation of an interleukin-I-like factor from human joint effusions. Arthritis Rheum 1983, 26:975-83.

8. Wisniewski KE, Dalton AJ, McLachlan C, Wen GY, Wisniewski HM: Alzheimer's disease in Down's syndrome: clinicopathologic studies. Neurology 1985, 35:957-61.

FI000 factor 8

Evaluated by W Sue Griffin 18 Nov 2011

9. Tanzi RE, Gusella JF, Watkins PC, Bruns GA, St George-Hyslop P, Van Keuren ML, Patterson D, Pagan S, Kurnit DM, Neve RL: Amyloid beta protein gene: cDNA, mRNA distribution, and genetic linkage near the Alzheimer locus. Science 1987, 235:880-4.

FI000 factor 6

Evaluated by W Sue Griffin 18 Nov 2011

10. Sheng JG, Mrak RE, Griffin WS: S100 beta protein expression in Alzheimer disease: potential role in the pathogenesis of neuritic plaques. J Neurosci Res 1994, 39:398-404. 
II. Griffin WS, Sheng JG, Roberts GW, Mrak RE: Interleukin-I expression in different plaque types in Alzheimer's disease: significance in plaque evolution. J Neuropathol Exp Neurol 1995, 54:276-8I.

12. Mrak RE, Sheng JG, Griffin WS: Correlation of astrocytic SI 00 beta expression with dystrophic neurites in amyloid plaques of Alzheimer's disease. J Neuropathol Exp Neurol 1996, 55:273-9.

13. Giulian D, Baker TJ, Shih LC, Lachman LB: Interleukin I of the central nervous system is produced by ameboid microglia. J Exp Med 1986, 164:594-604.

FI000 factor 6

Evaluated by W Sue Griffin 18 Nov 201I

14. Giulian D, Young DG, Woodward J, Brown DC, Lachman LB: Interleukin- $I$ is an astroglial growth factor in the developing brain. J Neurosci 1988, 8:709-14.

FI000 factor 6

Evaluated by W Sue Griffin 18 Nov 2011

15. Kligman D, Marshak DR: Purification and characterization of a neurite extension factor from bovine brain. Proc Natl Acad Sci U S A 1985, 82:7136-9.

FI000 factor 6

Evaluated by W Sue Griffin 18 Nov 2011

16. Goldgaber D, Harris HW, Hla T, Maciag T, Donnelly RJ, Jacobsen JS, Vitek MP, Gajdusek DC: Interleukin I regulates synthesis of amyloid beta-protein precursor mRNA in human endothelial cells. Proc Natl Acad Sci U S A 1989, 86:7606-10.

17. Griffin WS, Stanley LC, Ling C, White L, MacLeod V, Perrot LJ, White CL 3rd, Araoz C: Brain interleukin I and S- 100 immunoreactivity are elevated in Down syndrome and Alzheimer disease. Proc Natl Acad Sci U S A 1989, 86:76I I-5.
18. Li Y, Liu L, Barger SW, Griffin WS: Interleukin-I mediates pathological effects of microglia on tau phosphorylation and on synaptophysin synthesis in cortical neurons through a p38MAPK pathway. J Neurosci 2003, 23: I605-II.

19. Griffin WS, Liu L, Li Y, Mrak RE, Barger SW: Interleukin-I mediates Alzheimer and Lewy body pathologies. J Neuroinflammation 2006, 3:5.

20. Li Y, Liu L, Kang J, Sheng JG, Barger SW, Mrak RE, Griffin WS: Neuronal-glial interactions mediated by interleukin-I enhance neuronal acetylcholinesterase activity and mRNA expression. J Neurosci 2000, 20:149-55.

21. Breitner JC, Gau BA, Welsh KA, Plassman BL, McDonald WM, Helms MJ, Anthony JC: Inverse association of anti-inflammatory treatments and Alzheimer's disease: initial results of a cotwin control study. Neurology 1994, 44:227-32.

FI000 factor 8

Evaluated by W Sue Griffin 18 Nov 20II

22. Vlad SC, Miller DR, Kowall NW, Felson DT: Protective effects of NSAIDs on the development of Alzheimer disease. Neurology 2008, 70:1672-7.

FI000 factor 8

Evaluated by W Sue Griffin 18 Nov 20II

23. Breitner JC, Baker LD, Montine TJ, Meinert CL, Lyketsos CG Ashe KH, Brandt J, Craft S, Evans DE, Green RC, Ismail MS, Martin BK, Mullan MJ, Sabbagh M, Tariot PN; ADAPT Research Group: Extended results of the Alzheimer's disease anti-inflammatory prevention trial. Alzheimers Dement 201 I, 7:402-II.

FIO00 factor 9

Evaluated by W Sue Griffin 18 Nov 20II, Charlotte Teunissen 25 Aug 2011 